\title{
Effect of FABP5 gene silencing on the proliferation, apoptosis and invasion of human gastric SGC-7901 cancer cells
}

\author{
GUANJIE ZHAO ${ }^{1,2}$, MINFEI WU ${ }^{3}$, XIAOFENG WANG ${ }^{4}$, ZHENWU DU $^{1,5}$ and GUIZHEN ZHANG ${ }^{1,5}$ \\ ${ }^{1}$ Research Center of The Second Hospital, Jilin University, Changchun, Jilin 130041; \\ ${ }^{2}$ Department of Nephropathy, China-Japan Union Hospital of Jilin University, Changchun, Jilin 130033; \\ ${ }^{3}$ Department of Orthopedics, The Second Hospital of Jilin University, Changchun, Jilin 130041; \\ ${ }^{4}$ Department of Stomatology, China-Japan Union Hospital of Jilin University, Changchun, Jilin 130033; \\ ${ }^{5}$ Orthopedics Institute of The Second Hospital, Jilin University, Changchun, Jilin 130041, P.R.China
}

Received December 6, 2016; Accepted July 10, 2017

DOI: $10.3892 / \mathrm{ol} .2017 .6748$

\begin{abstract}
The aim of the present study was to examine the effect of fatty acid binding protein-5 (FABP-5) gene on the proliferation, apoptosis and invasion of human gastric cancer SGC-7901 cells. The viability, apoptosis and cell invasion of SGC-7901 cells before and after FABP5 knockdown were taken as the study objects, design and synthesis of siRNA interference sequence were conducted according to FABP-5 mRNA coding sequences, and SGC-7901 cells were transiently transfected. The human gastric cancer SGC-7901 cells were divided into three groups: FABP-5 siRNA group, negative control group and blank control group. FABP-5 gene mRNA and protein expression levels were detected by RT-PCR and western blot analysis. The CCK-8 assay was used to detect in vitro cell proliferation, flow cytometry (FCM) was used to detect changes in cell cycle and apoptosis in each group, TUNEL staining was used to detect apoptosis in each group, and the cell invasion chamber assay was used to detect cell invasiveness in each group. Each test was repeated three times. The results of the RT-PCR and western blot analysis showed that, expression of FABP-5 mRNA and protein in the FABP-5 siRNA group was significantly decreased compared with the negative and blank control groups. The cell growth rate in the FABP-5 siRNA group was significantly retarded, cell cycle was arrested in G0/G1 phase, the number of cells in $\mathrm{S}$ phase was reduced, and compared with the negative and blank control groups, the apoptotic rate in the FABP-5 siRNA group was significantly increased $(\mathrm{P}<0.01)$, while proliferation and invasiveness were significantly decreased $(\mathrm{P}<0.05)$. In conclusion, specific $F A B P-5$ gene silencing may reduce the
\end{abstract}

Correspondence to: Dr Guizhen Zhang, Research Center of The Second Hospital, Jilin University, 218 Ziqiang Road, Changchun, Jilin 130041, P.R. China

E-mail: fujtgh@163.com

Key words: RNA interference, epidermal fatty acid binding protein 5, cell cycle, apoptosis, gastric cancer invasiveness of gastric cancer cells, inhibit cell proliferation, and arrest cell cycle in G0/G1 phase, resulting in a significant increase in apoptosis.

\section{Introduction}

Gastric cancer is one of the most common malignant tumors, and although chemotherapy and neoadjuvant chemotherapy have been widely used in the treatment of gastric cancer, the prognosis of gastric cancer is poor, especially for patients with clinical metastasis and tumor recurrence $(1,2)$. Epidermal-type fatty acid binding protein-5 (FABP-5) gene is a fatty acid binding protein found in epidermal cells. It is a key molecule in tumor development. In a variety of tumors, the tumor-associated epithelial cell adhesion molecule upregulates the expression of $F A B P-5$ gene. Tumor cells with a high expression of $F A B P-5$ gene can affect cell signal transduction function by fatty acid metabolites (3-6). At present, there is no relevant report regarding the role of $F A B P-5$ gene in gastric cancer. In order to further study the mechanism of $F A B P-5$ gene in gastric cancer, this study employed RNA interference technology to silence $F A B P-5$ gene in human gastric SGC-7901 cancer cells, and the effect on tumor cell proliferation, apoptosis and invasiveness was observed.

\section{Materials and methods}

Materials. The human gastric cancer cell line (SGC-7901) was purchased from Shanghai Ji Kai Gene Technology Co., Ltd. (Shanghai, China). shRNA target designed recombinant $F A B P-5$ gene silencing lentiviral particles LV-shRNA-FABP-5 and the control empty vector lentiviral particles (LV-shRNA-NC) were provided by the Shanghai Bio-engineering Co., Ltd. (Shanghai, China). DMEM, phosphate buffer (phosphatic buffered saline, PBS), and fetal bovine serum were purchased from Hyclone; GE Healthcare (Logan, UT, USA). TRIzol was purchased from Invitrogen; Thermo Fisher Scientific (Waltham, MA, USA) and the reverse transcription kit was purchased from Fermentas; Thermo Fisher Scientific (Waltham, MA, USA). A fluorescence quantitative PCR kit was purchased from Takara Biotechnology Co., Ltd. 
(Dalian, China); DNA marker was purchased from Guangzhou Dongsheng Biotech Co., Ltd. (Guangzhou, China); western blot analysis and IP cell lysate, phenylmethylsulfonyl (phenylmethanesulfonyl fluoride, PMSF), loading buffer (5X) on SDS-PAGE protein, BCA protein concentration of the test kit (Enhanced), 20X TBS buffer were purchased from Jiangsu Pik days Biotechnology Research Institute (Nanjing, China); PVDF membrane was purchased from Merck Millipore (Billerica, MA, USA); propidium iodide (PI) and RNase enzymes were purchased from Fermentas; Thermo Fisher Scientific; flow cytometry was purchased from BD Biosciences (Franklin Lakes, NJ, USA); fluorescence microscope was purchased from Olympus Corp. (Tokyo, Japan); apoptosis kit was purchased from eBioscience company (Vienna, Austria); and the cell invasion assay was purchased from cell Invasion Assay Kit (Chemicon International (Billerica, MA, USA); cat. no. ECM550). The primers for the FABP-5 and GAPDH gene sequence were produced and verified by Shanghai Bio-engineering Co. Ltd., the same to a previous report (5). Rabbit anti-human FABP-5 monoclonal antibody was purchased from Abcam (Cambridge, UK); and mouse anti-human GAPDH monoclonal antibodies were purchased from Beijing Zhongshan Golden Bridge Biotechnology Co. Ltd. (Beijing, China).

Methods. Cell culture and transfection. Gastric cancer SGC-7901 cells were cultured with DMEM medium containing $10 \%$ fetal calf serum and $100 \mathrm{U} / \mathrm{ml}$ levofloxacin in a sealed incubator $\left(37^{\circ} \mathrm{C}, 5 \% \mathrm{CO}_{2}\right)$. Cells in the logarithmic growth phase were randomly selected: the FABP-5-shRNA group was treated with Lv-shRNA-FABP-5, the negative control group was treated with (LV-shRNA-NC), and the control group was routinely cultured. At $12 \mathrm{~h}$ before transfection, human gastric cancer SGC-7901 cells in the logarithmic phase were digested with trypsin and prepared into cell suspension. The cells were seeded in 6 -well plates $\left(5 \times 10^{4}\right)$ and cultured in a $37^{\circ} \mathrm{C}, 50 \mathrm{ml} / 1 \mathrm{CO}_{2}$ incubator until cell confluence was up to $20-30 \%$. Transfection was performed, and polybrene and infection enhancement solution were added to each well, with multiplicity of infection (MOI) being 10. Three replicate wells were set for each group. The LV-shRNA-FABP-5 target sequence was 5'-TGGGAAGGAAAGCACAATA-3', while the target sequence for the $\mathrm{NC}$ (negative control) was 5'-TTCTCCGAACGTGTCACGT-3'. The cells were harvested 3 days after transfection. The same amount of medium instead of the transfection system was used for the blank control group. Cells continued to be cultured in the $37^{\circ} \mathrm{C}, 5 \% \mathrm{CO}_{2}$ humidified incubator.

RT-PCR to detect FABP-5 mRNA expression. At $72 \mathrm{~h}$ after transfection, total RNA of SGC-7901 cells $\left(4 \times 10^{5}\right.$ cells) was extracted using the TRIzol kit. Quantitation cDNA was then synthesized according to the reverse transcription kit protocol. Primer 5.0 software was used to design primers as follows: FABP-5 upstream primer: 5'-TGAAGGAGCTAGGAGTGGGAA-3', downstream primer: 5'-TGCACCATCTGTAAAGTTGCAG-3', amplified fragment $212 \mathrm{bp}$; internal reference GAPDH upstream primer: 5'-TGACTTCAACAGCGACACCCA-3' downstream primers: 5'-CACCCTGTTGCTGTAGCCAAA-3', amplified fragment
$121 \mathrm{bp}$. Total RNA of five groups of cells was extracted using TRIzol reagent and reverse transcribed to cDNA, after which three replicate wells were set for each group. PCR primers were designed and synthesized by Shanghai Sangon Biological Engineering Technology \& Services Co., Ltd. (Shanghai, China), and three replicate wells were set for each group. Each experiment was repeated three times.

The RT-PCR reaction conditions used were: $95^{\circ} \mathrm{C}$ denaturation for $5 \mathrm{~min}, 95^{\circ} \mathrm{C}$ denaturation for $30 \mathrm{sec}, 60^{\circ} \mathrm{C}$ annealing for $30 \mathrm{sec}, 72^{\circ} \mathrm{C}$ extension for $60 \mathrm{sec}$, a total of 40 cycles. PCR products were subjected to $1.2 \%$ agarose gel electrophoresis, and a gel imager was used to observe results. The UVI gel imaging system was used to capture pictures. Image-Pro Plus 7.0 software was used to analyze the gray values, with the FABP-5/GAPDH ratio as the FABP-5 mRNA relative expression level.

Western blot analysis to detect FABP-5 protein expression. At $72 \mathrm{~h}$ after transfection, the total protein of cells was extracted in each experimental group and the protein concentration was determined using the BCA kit. Total protein $(50 \mu \mathrm{g})$ was subjected to $8 \%$ SDS-polyacrylamide gel electrophoresis (SDS-PAGE) and transferred to PVDF membranes by the wet transfer method. After film transfer, the membrane was blocked with $10 \%$ non-fat dry milk at room temperature for $2 \mathrm{~h}$. Subsequently, rabbit polyclonal FABP-5 antibody (dilution, 1/500; cat. no. ab37267) and rabbit polyclonal GAPDH antiboody (dilution, 1:500, cat. no. ab37168) purchased from Abcam (Cambridge, MA, USA) were added. They were placed on a shaker and incubated overnight at $4^{\circ} \mathrm{C}$. The next day, the membrane was washed by (triethanolamine buffered saline solution) TBS-T three times for $10 \mathrm{~min}$ each time. Then secondary goat anti-rabbit (HRP) IgG antibody (dilution, 1:2,000 (Abcam); cat. no. ab6721) was added after washing the membrane. The membranes were then exposed in the dark. Ultra-sensitive ECL chemiluminescence was used to detect protein bands, images were captured and striped gray value analyzed. The relative expression of the target protein was calculated as: gray value of target protein bands/gray value of internal reference protein bands.

CCK8 assay to detect SGC-7901 cell proliferation. SGC-7901 cells were seeded in 96-well culture plates at 4x103/well and cultured with $200 \mu$ l DMEM medium containing $10 \%$ fetal calf serum. Each group set five wells and separate blank wells were set as the control. CCK-8 $(20 \mu \mathrm{l})$ was added into each well. After incubation for $4 \mathrm{~h}$, the absorbance at $490 \mathrm{~nm}$ was detected by a microplate reader, the average of five wells was calculated, and the growth curve was drawn.

Transwell chamber invasion assay. The polycarbonate membrane filter was capped with $50 \mu \mathrm{g}$ Matrigel per well in a well-polymerized lower chamber, after which $10 \%$ fetal bovine serum was added as conditioned medium. Then, $100 \mu 1$ SGC-7901 cell suspension of the three groups $\left(3 \times 10^{5} / 1\right)$ was added in the upper chamber, placed in an incubator for $24 \mathrm{~h}$ and fixed with $4 \%$ paraformaldehyde for $10 \mathrm{~min}$ prior to staining with hematoxylin for $20 \mathrm{~min}$. The cells on the lower surface of membrane were counted under a light microscope (BX-42, Olympus Corp.). Penetrating cells in five random fields 
A

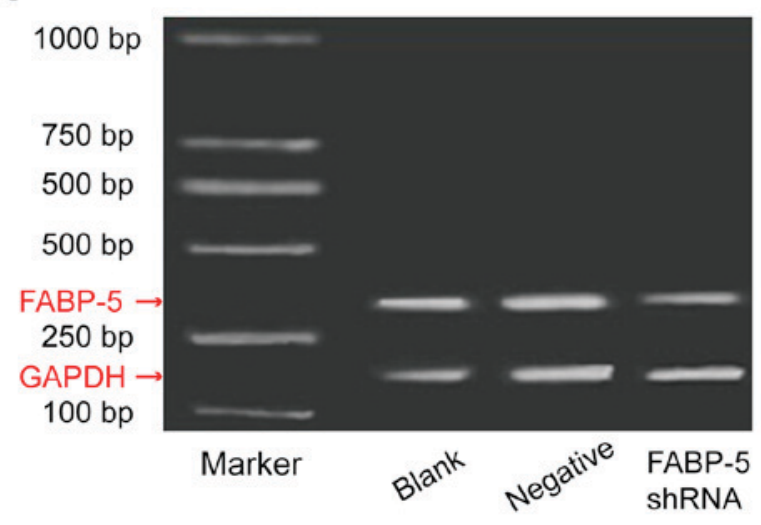

B

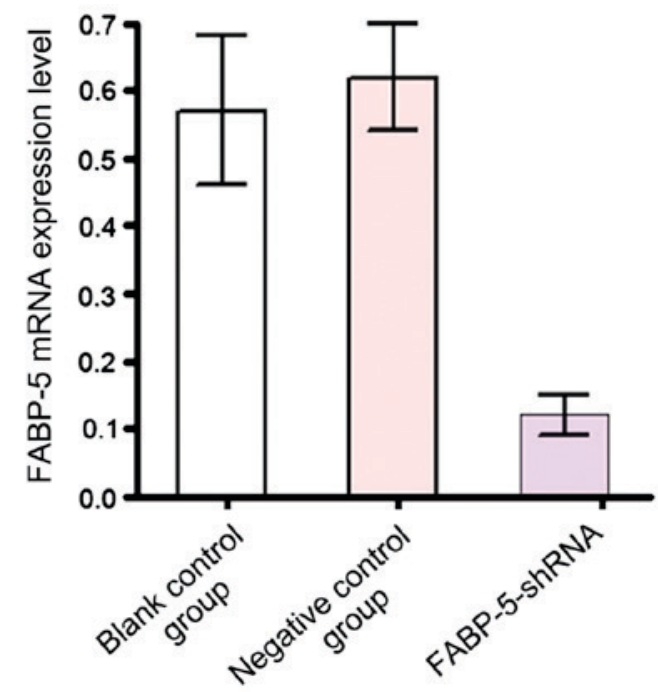

Figure 1. shRNA downregulated FABP-5 mRNA expression level in SGC-7901 cells. (A) FABP-5 mRNA expression in SGC7901 cells before and after FABP5 shRNA transfection was determined by RT-PCR. (B) FABP-5 mRNA expression level was determined by quantitative RT-PCR.

were counted for each film, and the average was calculated. Each group set three chambers in parallel. Experiments were repeated three times. The cell invasion rate (\%) was calculated as the total number of penetrating cells/total number of cells seeded in the upper chamber $\mathrm{x} 100 \%$.

Cell cycle detection. Three groups of cells were trypsinized and washed with PBS twice and fixed with $1 \mathrm{ml}$ pre-chilled $70 \%$ ethanol at $4^{\circ} \mathrm{C}$ overnight. The cells were washed with PBS twice and measured for $1.0 \times 10^{5} / \mathrm{ml}$ cell suspension. After mixing, an appropriate amount of PI solution (cell suspension, $\mathrm{PI}=1: 1$ ) was added. The cells were incubated for $30 \mathrm{~min}$ at $4^{\circ} \mathrm{C}$ in the dark. A 300 mesh screen filter was used to filter the cell suspension and remove adhesion cells. Flow cytometry was used to analyze DNA content, and the software was used to analyze the cell number in $\mathrm{G} 0 / \mathrm{G} 1, \mathrm{~S}, \mathrm{G} 2 / \mathrm{M}$ phases and the proportion.

Cell apoptosis detected by flow cytometry. At $72 \mathrm{~h}$ after transfection, the cells in each group were collected and digested by EDTA-free trypsin. Then they were transferred into a $1.5 \mathrm{ml}$ sterile Eppendorf tube. The centrifugation was performed at $4^{\circ} \mathrm{C}, 1,650 \mathrm{x} \mathrm{g}$ for $5 \mathrm{~min}$. The supernatant was discarded and the cells were washed with PBS twice, and $500 \mu$ l binding buffer was added to re-suspend cells. Annexin V-FITC $(5 \mu \mathrm{l})$ and PI $(5 \mu \mathrm{l})$ were added at room temperature in the dark. After reacting for 5-15 min, the cells were detected by flow cytometry within $1 \mathrm{~h}$. The excitation wavelength (Ex) was $488 \mathrm{~nm}$, and the emission wavelength (Em) was $530 \mathrm{~nm}$.

TUNEL staining. At $72 \mathrm{~h}$ after grouping, the cells in each group were collected and MGC-803 glass slides were prepared. After aspirating the cultured medium, the cells were air dried, fixed with 4\% paraformaldehyde, treated with fresh 3\% H202 at room temperature. Then, $0.1 \%$ Triton X-100 (dissolved in $0.1 \%$ sodium citrate solution) was used for drilling. According to the TUNEL kit instructions, DAB staining and hematoxylin re-staining were performed. This was followed by gradient ethanol dehydration, xylene transparency and neutral gum sealing, after which the samples were observed under the microscope. Three horizons were observed in each slice, and 300 consecutive cells were counted in each field. The percentage of apoptotic cells was the apoptosis index (m), and was calculated as: AI $(\%)=$ number of apoptotic cells/total number of cells x $100 \%$.

Statistical analysis. SPSS 16.0 statistical software (Chicago, IL, USA) was used for analysis. Apoptosis data of the two groups were compared using the t-test, and one-way ANOVA was used to determine the remaining measurement data among groups. Experimental data were presented as mean \pm SD. $\mathrm{P}<0.05$ was considered statistically significant.

\section{Results}

shRNA downregulated FABP-5 mRNA expression level in SGC-7901 cells. After RNA interference, RT-PCR results showed the FABP-5 mRNA level in SGC-7901 cells (Fig. 1A and B). The relative expression level $(0.12 \pm 0.03)$ was significantly reduced in the FABP-5-shRNA group compared with the negative control group $(0.62 \pm 0.08 \%)$ and blank control group $(0.57 \pm 0.11 \%)(\mathrm{P}<0.01)$ and the inhibition rate was $(70.27 \pm 1.38 \%)$. There was no significant difference in FABP-5 mRNA expression between the negative and blank control groups, suggesting that the interference sequence designed and produced in this study can specifically inhibit the expression of FABP-5 gene (Fig. 1).

shRNA down-regulated FABP-5 protein expression level in SGC-7901 cells. Western blot analysis revealed, after RNA interference, the FABP-5 protein level in SGC-7901 cells (Fig. 2A and B). The relative expression level $(0.32 \pm 0.03)$ was significantly reduced in the FABP-5-shRNA group compared with the negative control group (1.62 \pm 0.12$)$ and blank control group $(1.57 \pm 0.14)(\mathrm{P}<0.01)$. The software Image $\mathrm{J}$ was used to analyze the gray values of bands and calculate the inhibition rate, which was $(72.56 \pm 1.24 \%)$. By contrast, there was no significant difference in FABP-5 protein expression 
A

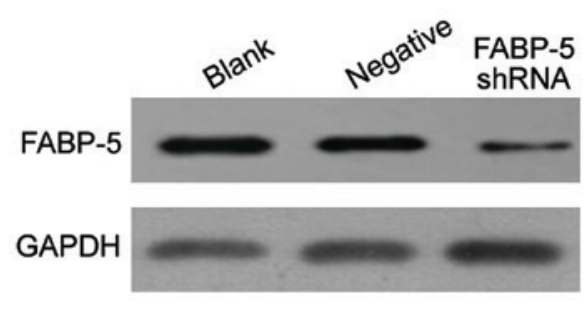

B

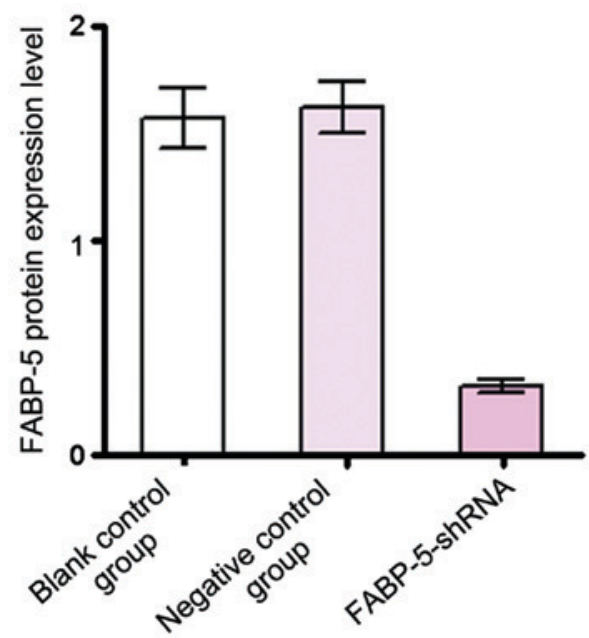

Figure 2. shRNA downregulated FABP-5 protein expression level in SGC-7901 cells. (A) FABP-5 protein expression in SGC-7901 cells was examined. (B) Quantitative analysis of FABP-5 protein expression levels in the three groups. FABP-5, fatty acid binding protein-5.

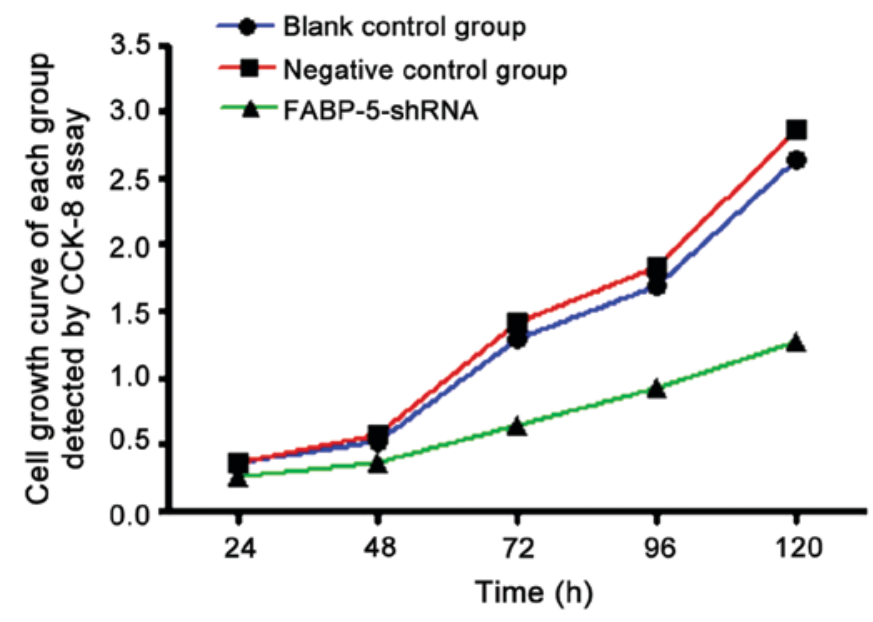

Figure 3. Cell proliferation of SGC-7901 cells was detected by CCK-8 assay.

between the negative and blank control groups, indicating that the interference sequence designed and produced in this study can specifically inhibit the expression of FABP-5 protein (Fig. 2).

CCK-8 assay to detect reduced SGC-7901 cell proliferation in FABP-5-shRNA group. CCK-8 test results showed that compared with the blank and negative control groups, A values at $490 \mathrm{~nm}$ in the FABP-5-shRNA group were lower at 24, 48, 72,96 and $120 \mathrm{~h}$ after transfection, and the differences were statistically significant $(P<0.05)$. The growth curve showed that, the curve of FABP-5-shRNA group was significantly lower than that of the blank and negative control groups, and the difference was statistically significant $(\mathrm{P}<0.05)$. It indicated that the cell proliferation in the FABP-5-shRNA group was significantly inhibited (Fig. 3; Table I).

Cell invasiveness was reduced in the FABP5-siRNA group. As shown in Fig. 4, the number of cells identified across the membrane in the blank and negative control groups were higher
[(59.22 \pm 6.32$)$ and (61.27 \pm 7.36$)]$, the number of cells across the membrane in the FABP-5-shRNA group was significantly reduced (28.46 \pm 4.58$)$, and the difference was statistically significant $(\mathrm{P}<0.01)$. The results showed that specifically interfering with $F A B P-5$ gene expression effectively reduces the invasiveness of SGC-7901 cells (Fig. 4).

Flow cytometry to detect cell cycle. The proportion of cells in G1 phase in the FABP-5-shRNA group was reduced compared to the blank and negative control groups $(\mathrm{P}<0.05)$. Proportions of S-phase cells and G2/M-phase cells in the FABP-5-shRNA group increased compared with the blank and negative control groups (all $\mathrm{P}<0.05$ ). In the FABP-5-shRNA group, cells in G1 phase decreased, and cells in S-phase and $\mathrm{G} 2 / \mathrm{M}$ phase increased in the negative and blank control groups, and the difference was not statistically significant (P>0.05) (Table II; Fig. 5).

Apoptotic cells detected by flow cytometry. In the DNA histogram of flow cytometry the SGC-7901 cell apoptosis rate of the FABP-5-shRNA group $(4.76 \pm 0.16 \%)$ was significantly higher than that of the blank control group $(2.13 \pm 0.36 \%)$ and the negative control group $(2.13 \pm 0.36 \%)$, and the difference was statistically significant $(\mathrm{P}<0.05)$ (Fig. 6). RNA interference to silence $F A B P-5$ gene can significantly promote apoptosis of SGC-7901 cells.

TUNEL staining to observe apoptosis. In the FABP-5-shRNA group, there were apoptotic cells with brown-stained nuclei (Fig. 7, arrow). A comparison of the negative and blank control groups showed the results for the apoptotic index were $(5.86 \pm 1.23 \%),(6.26 \pm 1.75 \%)$ and $(38.64 \pm 6.84 \%)$, and the difference was significant $(\mathrm{P}<0.01)$.

\section{Discussion}

FABPs are a group of small and highly-conserved cytoplasmic proteins, widely expressed in a variety of mammal cells, and which can bind to long-chain fatty acid cytoplasmic protein, 
Table I. Comparison of cell viability of three groups of cells at different time points ${ }^{\mathrm{a}}$.

\begin{tabular}{lccccc}
\hline Groups & $24 \mathrm{~h}$ & $48 \mathrm{~h}$ & $72 \mathrm{~h}$ & $96 \mathrm{~h}$ & $120 \mathrm{~h}$ \\
\hline Blank control group & $0.36 \pm 0.03$ & $0.55 \pm 0.04$ & $1.36 \pm 0.11$ & $1.79 \pm 0.18$ & $2.74 \pm 0.21$ \\
Negative control group & $0.35 \pm 0.02$ & $0.56 \pm 0.06$ & $1.40 \pm 0.13$ & $1.81 \pm 0.16$ & $2.75 \pm 0.19$ \\
FABP-5-shRNA group & $0.26 \pm 0.02$ & $0.36 \pm 0.04$ & $0.64 \pm 0.09$ & $0.92 \pm 0.12$ & $1.28 \pm 0.17$ \\
\hline
\end{tabular}

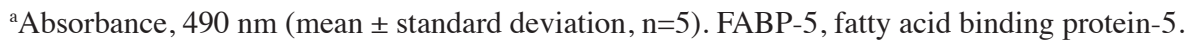
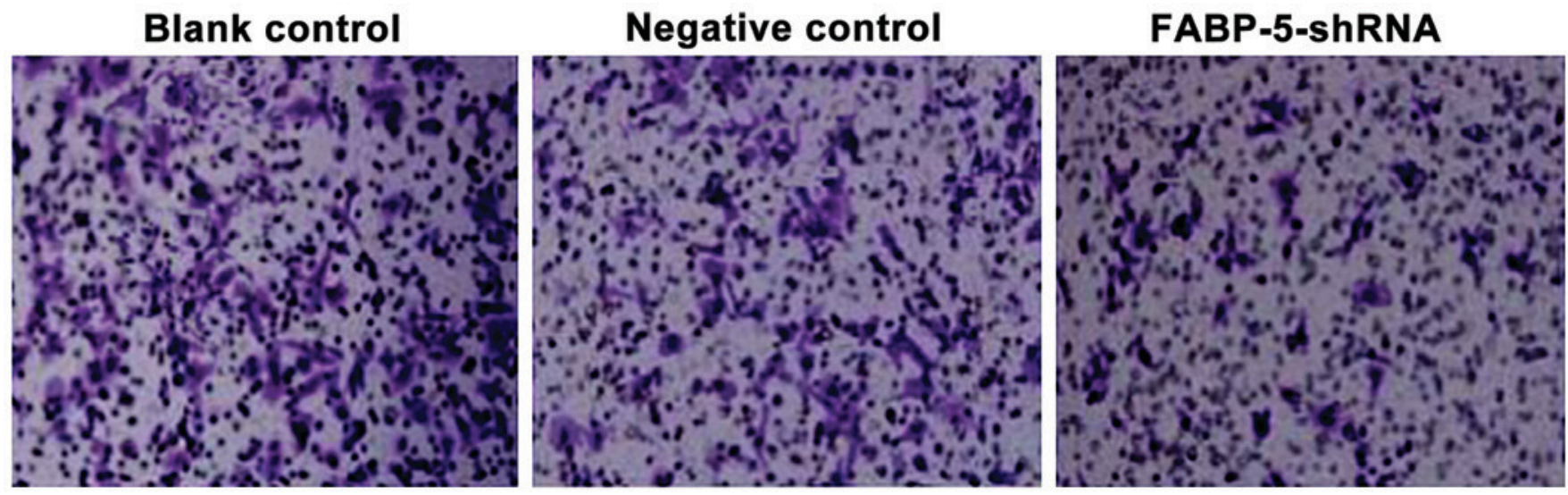

Figure 4. FABP-5-shRNA inhibited cell invasion in SGC7901 cells. FABP-5, fatty acid binding protein-5.

Table II. Cell cycle distribution and apoptosis rate (\%).

\begin{tabular}{lccc}
\hline Groups & G1 & S & G2/M \\
\hline $\begin{array}{l}\text { Blank } \\
\text { control group }\end{array}$ & $62.83 \pm 0.84$ & $30.56 \pm 0.64$ & $7.53 \pm 0.56$ \\
$\begin{array}{l}\text { Negative } \\
\text { control group }\end{array}$ & $63.46 \pm 0.79$ & $29.17 \pm 0.46$ & $7.49 \pm 0.73$ \\
$\begin{array}{l}\text { FABP-5-shRNA } \\
\text { group }\end{array}$ & $32.75 \pm 0.56$ & $48.34 \pm 0.96$ & $18.84 \pm 0.57$ \\
\hline
\end{tabular}

FABP-5, fatty acid binding protein-5.

playing an important role in the uptake, transport and metabolism of long-chain fatty acids (7). FABPs are tissue-specific, named by the tissue from which they were initially isolated or identified. Currently, there are nine types of FABP, including the epidermal-relevant type FABP and myocardium-type FABP. The most basic function of FABPs is to be involved in the intake and intracellular transport of fatty acids (8). Expression of FABPs is increased in many types of cancer. FABPs affect tumor growth and progression by combining with transported fatty acids and their derivatives, hormones, steroids, carcinogens and other ligands $(9,10)$. Recently, an increasing number of studies have shown that FABPs are expressed in different degrees in most malignancies, including breast, prostate, liver, lung and bladder epithelium cancer, and are associated with the incidence, metastasis, invasion, poor prognosis and resistance of malignant tumors (11-15).
FABP-5 is epidermal-relevant type, and the current study found that FABP-5 expressed by cells can combine with long-chain fatty acids, provide energy and raw materials for cell growth and participate in tumor growth-associated signal transduction. Fatty acid binding proteins are closely associated with tumors and a variety of other diseases $(9,16)$. In breast cancer (17) and lung squamous cell carcinoma (18), $F A B P-5$ gene was significantly upregulated to promote tumorigenesis. In primary NSCLC tissues, FABP-5 expression was associated with tumor grade and metastasis. The larger the tumor volume and the higher the tumor grade, the higher the expression of FABP-5, including patients with metastasis (19). Celis et al found that the FABP-5 expression level was positively correlated with the degree of differentiation of bladder cancer (20). Additionally, the expression level of FABP-5 decreased as the degree of differentiation of bladder cancer decreased (20). In prostate cancer, in vivo experiments confirmed that FABP5 downregulation can reduce tumor cell metastasis and inhibit tumor growth $(21,22)$. In intrahepatic bile duct cell carcinoma and squamous cell carcinoma, FABP-5 can promote the proliferation of tumor cells and enhance the invasion ability of cells $(23,24)$. Zhou et al transfected FABP-5-shRNA expression vector into human HepG2 cells and found that FABP-5-shRNA can significantly promote tumor cell apoptosis, arrest the cell cycle in G2/M phase to inhibit the proliferation of liver cancer cells, and reduce the invasiveness of liver cancer cells (6). In addition, head and neck cancer (25), endometrial cancer (26) and melanoma (27) are closely associated with the expression of FABP-5. A previous study on pathological tissues of esophageal cancer showed that FABP-5 gene expression was significantly increased, 

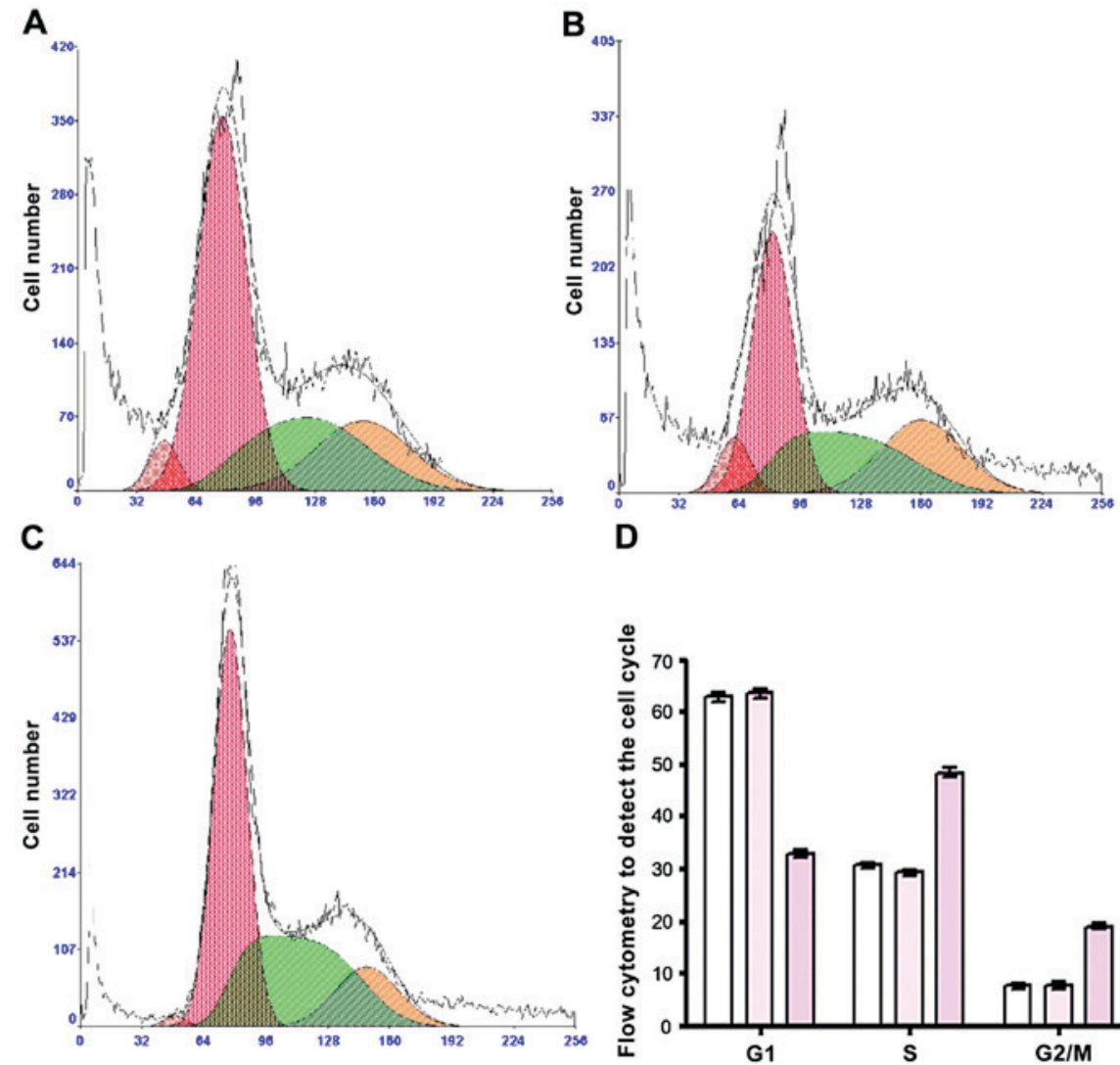

D

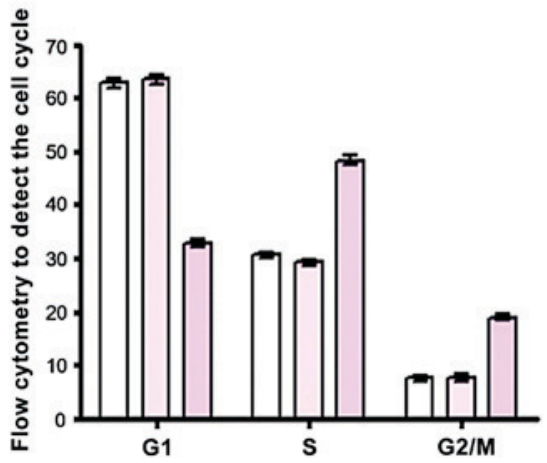

Figure 5. Flow cytometry was used to detect the cell cycle distribution. (A) Blank control group; (B) Negative control group; (C) FABP-5-shRNA group; (D) Quantitative analysis showed that the proportion of G1 phase cells in the FABP-5-shRNA group was reduced, while the proportions of cells in S phase and G2/M phase increased. FABP-5, fatty acid binding protein-5.
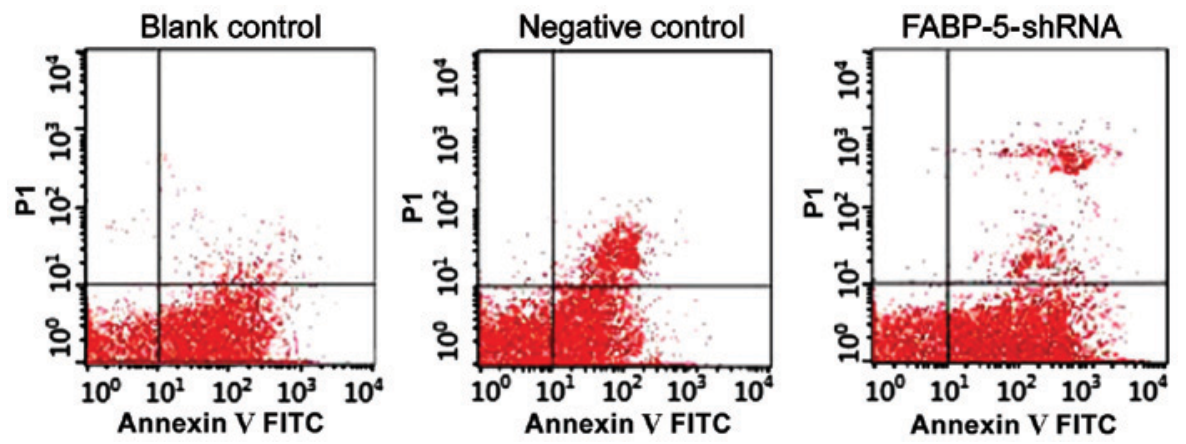

Figure 6. FA-C flow cytometry was performed to detect apoptosis in each group. FABP-5 gene silencing can significantly promote apoptosis of EC9706 cells. FABP-5 gene, fatty acid binding protein-5 gene.
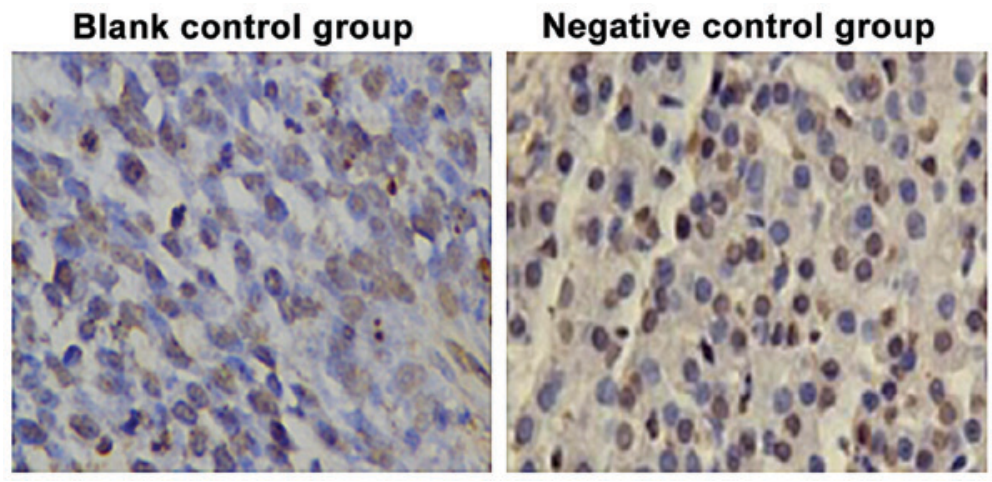

\section{FABP-5-shRNA group}

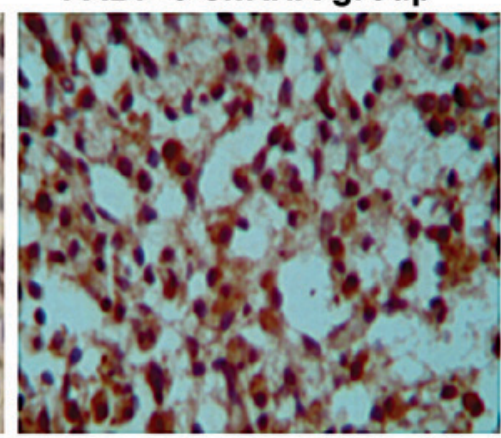

Figure 7. Cell apoptosis was detected by TUNEL staining. FABP-5-shRNA significantly promoted cell apoptosis, compared with the negative and blank control groups. FABP-5, fatty acid binding protein-5. 
suggesting that the upregulation of $F A B P-5$ gene expression may contribute to the development of esophageal cancer (28).

In the present study, we transfected FABP-5-shRNA expression vector into human gastric SGC-7901 cancer cells, and found that the relative expression levels of FABP-5 gene and protein in the FABP-5-shRNA group were significantly lower than those in the negative and blank control groups, indicating that the interference sequence designed and synthesized in this study can specifically inhibit the expression of FABP-5 gene. CCK-8 detection results showed that compared to the blank and negative control groups, cell proliferation in the FABP-5-shRNA group was significantly inhibited. Flow cytometry and TUNEL staining showed that FABP-5 gene silencing can significantly promote SGC-7901 cell apoptosis. Flow cytometry showed that after FABP-5 gene silencing, the SGC-7901 cell cycle was arrested in G2/M phase, and the proliferation of SGC-7901 cells was inhibited. The cell invasion chamber assay showed that cell invasiveness in the FABP-5-shRNA group was significantly lower than that in the blank and negative control groups, suggesting that $F A B P-5$ gene silencing reduced the invasiveness of gastric cancer cells.

In summary, using the lentivirus RNA interference to knockout FABP-5 gene can influence the proliferation of gastric cancer cells and induce apoptosis, and can inhibit the invasiveness of gastric cancer cells. These indicated that FABP-5 gene may be directly or indirectly involved in the cell cycle regulation and apoptosis of gastric cancer cells. These changes of the gene expression levels were closely associated with tumor cell invasiveness. Therefore, FABP-5 gene may become a target for the treatment of gastric cancer.

\section{References}

1. Zhu YL, Yang L, Sui ZQ, Liu L and Du JF: Clinicopathological features and prognosis of Borrmann type IV gastric cancer. J BUON 21: 1471-1475, 2016.

2. Yildiz B, Etiz D, Dal P, Junushova B, Pasaoglu O, Yilmaz E, Erkasap S and Dincer M: Tumor deposits: Prognostic significance in gastric cancer patients. J BUON 21: 1476-1481, 2016.

3. Veerkamp JH, Maatman RG and Prinsen CF: Fatty acid-binding proteins: Structural and functional diversity. Biochem Soc Trans 20: 801-805, 1992.

4. Münz M, Zeidler R and Gires O: The tumour-associated antigen EpCAM upregulates the fatty acid binding protein E-FABP. Cancer Lett 225: 151-157, 2005.

5. Uma RS, Naresh KN, D'Cruz AK, Mulherkar R and Borges AM: Metastasis of squamous cell carcinoma of the oral tongue is associated with down-regulation of epidermal fatty acid binding protein (E-FABP). Oral Oncol 43: 27-32, 2007.

6. Liu RZ, Graham K, Glubrecht DD, Germain DR, Mackey JR and Godbout R: Association of FABP5 expression with poor survival in triple-negative breast cancer: implication for retinoic acid therapy. Am J Pathol 178: 997-1008, 2011.

7. Smith S, Witkowski A and Joshi AK. Structural and functional organization of the animal fatty acid synthase. Prog Lipid Res 42: 289-317, 2003.

8. Chmurzyńska A: The multigene family of fatty acid-binding proteins (FABPs): Function, structure and polymorphism. J Appl Genet 47: 39-48, 2006.

9. Thumser AE, Moore JB and Plant NJ: Fatty acid binding proteins: Tissue-specific functions in health and disease. Curr Opin Clin Nutr Metab Care 17: 124-129, 2014.

10. Zimmerman AW, van Moerkerk HT and Veerkamp JH: Ligand specificity and conformational stability of human fatty acid-binding proteins. Int J Biochem Cell Biol 33: 865-876, 2001.
11. Kawamura T, Kanno R, Fujii H and Suzuki T: Expression of liver-type fatty-acid-binding protein, fatty acid synthase and vascular endothelial growth factor in human lung carcinoma. Pathobiology 72: 233-240, 2005.

12. Lawrie LC, Dundas SR, Curran S and Murray GI: Liver fatty acid binding protein expression in colorectal neoplasia. Br J Cancer 90: 1955-1960, 2004.

13. Hammamieh R, Chakraborty N, Barmada M, Das R and Jett M: Expression patterns of fatty acid binding proteins in breast cancer cells. J Exp Ther Oncol 5: 133-143, 2005.

14. Tölle A, Suhail S, Jung M, Jung K and Stephan C: Fatty acid binding proteins (FABPs) in prostate, bladder and kidney cancer cell lines and the use of IL-FABP as survival predictor in patients with renal cell carcinoma. BMC Cancer 11: 302, 2011.

15. Hashimoto T, Kusakabe T, Watanabe K, Sugino T, Fukuda T, Nashimoto A, Honma K, Sato Y, Kimura H, Fujii H, et al: Liver-type fatty acid-binding protein is highly expressed in intestinal metaplasia and in a subset of carcinomas of the stomach without association with the fatty acid synthase status in the carcinoma. Pathobiology 71: 115-122, 2004.

16. Armstrong EH, Goswami D, Griffin PR, Noy N and Ortlund EA: Structural basis for ligand regulation of the fatty acid-binding protein 5 , peroxisome proliferator-activated receptor $\beta / \delta$ (FABP5-PPAR $\beta / \delta$ ) signaling pathway. J Biol Chem 289: 14941-14954, 2014

17. Levi L, Lobo G, Doud MK, von Lintig J, Seachrist D, Tochtrop GP and Noy N: Genetic ablation of the fatty acid-binding protein FABP5 suppresses HER2-induced mammary tumorigenesis. Cancer Res 73: 4770-4780, 2013.

18. Harris FT, Rahman SM, Hassanein M, Qian J, Hoeksema MD, Chen H, Eisenberg R, Chaurand P, Caprioli RM and Shiota M, et al: Acyl-coenzyme A-binding protein regulates Beta-oxidation required for growth and survival of non-small cell lung cancer. Cancer Prev Res (Phila) 7: 748-757, 2014.

19. Liu Q, Wang S, Xu H and Zhang S: Expressions and significances of CRABPII and E-FABP in non-small cell lung cancer. Zhongguo Fei Ai Za Zhi 16: 12-19, 2013 (In Chinese).

20. Celis JE, Rasmussen HH, Vorum H, Madsen P, Honoré B, Wolf H and Orntoft TF: Bladder squamous cell carcinomas express psoriasin and externalize it to the urine. J Urol 155: 2105-2112, 1996.

21. Adamson J, Morgan EA, Beesley C, Mei Y, Foster CS, Fujii H, Rudland PS, Smith PH and Ke Y: High-level expression of cutaneous fatty acid-binding protein in prostatic carcinomas and its effect on tumorigenicity. Oncogene 22: 2739-2749, 2003.

22. Morgan EA, Forootan SS, Adamson J, Foster CS, Fujii H, Igarashi M, Beesley C, Smith $\mathrm{PH}$ and $\mathrm{Ke} \mathrm{Y}$ : Expression of cutaneous fatty acid-binding protein (C-FABP) in prostate cancer: Potential prognostic marker and target for tumourigenicity-suppression. Int J Oncol 32: 767-775, 2008.

23. Jeong CY, Hah YS, Cho BI, Lee SM, Joo YT, Jung EJ, Jeong SH, Lee YJ, Choi SK, Ha WS, et al: Fatty acid-binding protein 5 promotes cell proliferation and invasion in human intrahepatic cholangiocarcinoma. Oncol Rep 28: 1283-1292, 2012.

24. Fang LY, Wong TY, Chiang WF and Chen YL: Fatty-acidbinding protein 5 promotes cell proliferation and invasion in oral squamous cell carcinoma. J Oral Pathol Med 39: 342-348, 2010.

25. Rauch J, Ahlemann M, Schaffrik M, Mack B, Ertongur S, Andratschke M, Zeidler R, Lang S and Gires O: Allogenic antibody-mediated identification of head and neck cancer antigens. Biochem Biophys Res Commun 323: 156-162, 2004.

26. Li Z, Huang C, Bai S, Pan X, Zhou R, Wei Y and Zhao X: Prognostic evaluation of epidermal fatty acid-binding protein and calcyphosine, two proteins implicated in endometrial cancer using a proteomic approach. Int J Cancer 123: 2377-2383, 2008.

27. Brouard MC, Saurat JH, Ghanem G and Siegenthaler G: Urinary excretion of epidermal-type fatty acid-binding protein and S100A7 protein in patients with cutaneous melanoma. Melanoma Res 12: 627-631, 2002.

28. Ogawa R, Ishiguro H, Kuwabara Y, Kimura M, Mitsui A, Mori Y, Mori R, Tomoda K, Katada T, Harada K, et al: Identification of candidate genes involved in the radiosensitivity of esophageal cancer cells by microarray analysis. Dis Esophagus 21: 288-297, 2008. 